\title{
Serum Uric Acid Levels Mediated Atrial Fibrillation in Patients with Hypertension
}

\section{Maimaitiaili Aizezi ${ }^{1}$, Abulaikemu Mahemuti ${ }^{2}$, Maimaiti Yisireyili ${ }^{3 *}$, Weimin Zhang ${ }^{1}$, Aziguli Alimujiang ${ }^{3}$, Yang Zhao ${ }^{3}$, Tingyu $\mathrm{Ma}^{3}$, Xinsheng Zhao ${ }^{1}$ and Zonggang Zhang ${ }^{1}$}

${ }^{1}$ Department of Cardiac Surgery, People's Hospital of Xinjiang Uygur Autonomous Region, Urumqi, Xinjiang, P.R. China

${ }^{2}$ Department of Emergency and Traumatic Surgery, People's Hospital of Xinjiang Uygur Autonomous Region, Urumqi, Xinjiang, P.R. China

${ }^{3}$ Research Institute of General and Minimally Invasive Surgery, People's Hospital of Xinjiang Uygur Autonomous Region, Urumqi, Xinjiang, P.R. China

*Corresponding author: Maimaiti Yisireyili, Research Institute of General and Minimally Invasive Surgery, People's Hospital of Xinjiang Uygur Autonomous Region, Urumqi, Xinjiang, P.R. China, E-mail: maimaiti727@sina.com

Received: 04 Jun, 2019 | Accepted: 14 Jun, 2019 | Published: 19 Jun, 2019

Citation: Aizezi M, Mahemuti A, Yisireyili M, Zhang W, Alimujiang A, et al. (2019) Serum Uric Acid Levels Mediated Atrial Fibrillation in Patients with Hypertension. J Clin Case Stu 4(2): dx.doi.org/10.16966/2471-4925.188

Copyright: (C) 2019 Aizezi M, et al. This is an open-access article distributed under the terms of the Creative Commons Attribution License, which permits unrestricted use, distribution, and reproduction in any medium, provided the original author and source are credited.

\section{Abstract}

Background: Oxidative stress and inflammatory cytokines play an important role in the development of hypertension and Atrial Fibrillation (AF). Clinical studies have shown that serum Uric Acid (UA) levels are positively associated with hypertension and AF. Xanthine Oxidase (XO) is a key enzyme in UA metabolism. It can be a source of oxidative stress in AF. In this study, we investigated the role of XO in oxidative stress and inflammatory cytokines in UA-induced AF.

Methods: According to the inclusion and exclusion criteria, 60 patients hospitalized in the Xinjiang Uygur Autonomous Region People's Hospital from June 2017 to June 2018 were selected as the research group. Thirty healthy subjects who completed physical examination in the same period comprised the healthy control group. All subjects underwent echocardiography. Fasting blood samples were collected, and clinical data, such as sex, age, course of disease, BMI, smoking, drinking, dyslipidemia, diabetes mellitus, and diuretics, were recorded in detail. ELISA method was used to analyze the circulatory levels of UA, XO, nicotinamide adenine dinucleotide phosphate oxidase-4 (Nox-4; a biomarker of oxidative stress), and inflammatory cytokines (high-sensitivity C-reactive protein [Hs-CRP], interleukin-1 $[$ [IL-1 3 ], interleukin-8 [IL-8], and TNF- $\alpha$ ).

Results: According to the clinical manifestations, electrophysiological examination, and diagnostic criteria of hypertension, the patients were divided into hypertension with AF group (AF group), hypertension without AF group (non-AF group), and healthy control group. The average age, body mass index, course of hypertension, blood pressure, hyperlipidemia, and diabetes mellitus in the AF group were higher than in the non-AF and control groups $(\mathrm{P}<0.05)$. In comparison with the non-AF and control groups, the serum levels of high-density lipoprotein cholesterol, triglycerides, and creatinine in the AF group were significantly increased $(P<0.05)$, whereas the estimated glomerular filtration rate was significantly reduced $(P<0.05)$. In the $\mathrm{AF}$ group, the blood concentrations of $\mathrm{UA}, \mathrm{XO}$, and Nox-4 significantly increased compared with those in the non- $\mathrm{AF}$ and control groups $(P<0.001)$. The circulatory levels of inflammatory cytokines (Hs-CRP, IL- $1 \beta$, IL-8, and TNF- $\alpha$ ) significantly increased in the AF group (P<0.001). Pearson correlation analysis showed that the serum levels of $X O$ in the AF group have a significant positive correlation with the blood concentrations of Nox-4 and inflammatory cytokines (Hs-CRP, IL-1 $\beta$, IL-8, and TNF- $\alpha$ ) $(\mathrm{P}<0.01)$ with correlation coefficients of $r=0.99,0.91,0.96,0.95,0.97$.

Conclusions: XO showed remarkable elevated ROS accumulation and inflammatory cytokines, there by resulting in induced AF formation in patients with hypertension.

Keywords: Oxidative stress; Uric acid; Xanthine oxidase; Atrial fibrillation; Inflammatory cytokines

\section{Background}

Atrial Fibrillation (AF) is the most common persistent arrhythmia, and its incidence increases with age. Hypertension is a major risk factor of AF. The common arrhythmia in hypertensive patients is caused by the fact that AF and hypertension promote each other, thereby creating a vicious circle that accelerates the progression of hypertension [1-2]. The mechanism underlying their occurrence and development has not yet been clarified.
Serum Uric Acid (SUA) is an important plasma marker in patients with Coronary Heart Disease (CHD) and hypertension, which is closely related to the morbidity and mortality of Cardio Vascular Diseases (CVD) [3]. UA in the body is the final product of purine base metabolism. It is produced by hypoxanthine and xanthine via purine synthesis under the catalysis of Xanthine Oxidase (XO) [4]. The increased enzymatic activity of XO can lead to excessive accumulation of Reactive Oxygen Species (ROS), eventually causing atrial remodeling and promoting AF's occurrence [5]. The increase of 
SUA level reflects the increase of XO activity in mammals and humans [6]. ROS contributes to the pathological consequences of $\mathrm{AF}$ and hypertension [7]. AF is also associated with excessive ROS generation. The high concentrations of Nicotinamide Adenine Dinucleotide Phosphate (NADPH) oxidase-4 (Nox-4) is markedly correlated with acute myocardial infarction and AF [8]. Nox-4 is frequently used as a biomarker of oxidative stress [9]. However, the role Nox-4 in the occurrence of $\mathrm{AF}$ and hypertension has not been fully investigated.

Inflammation plays an important role in the development of AF. Inflammation has been implicated in the pathophysiology of AF and modulates the cell signaling activation patterns associated with fibrosis, apoptosis, and visceral hyper sensation [10]. The serum levels of tumor necrosis factor- $\alpha$ (TNF- $\alpha$ ) and interleukin-8 (IL-8), which are inflammatory mediators, remarkably increase in patients with $\mathrm{AF}$ [11]. Elevated serum levels of inflammatory biomarkers in subjects with AF and expression of inflammatory markers in cardiac tissues of patients with AF suggest the substantial role of inflammation in AF's pathogenesis [12]. Inflammation is linked to various pathological processes, such as oxidative stress, apoptosis, and fibrosis, which promote AF formation. The frequencies of AF episodes are substantially reduced and the symptoms of AF are ameliorated in patients with concomitant Hyperuricamia (HUA) after treatment with UA inhibitors [13].

Therefore, the effects of UA, ROS, and inflammatory cytokines on the occurrence of AF need further study. Our study aims to examine the expressions of UA, XO, ROS marker (Nox-4), and inflammatory cytokines (High-sensitivity C-reactive protein [Hs-CRP], IL-8, IL$1 \beta$, and TNF- $\alpha$ ) in the serum of patients with hypertension and AF. This work also aims to explore the correlations of XO-induced ROS production and inflammation and determine its influences on the occurrence of AF.

\section{Methods}

\section{Patient characteristics}

Sixty patients admitted to the Xinjiang Uygur Autonomous Region People's Hospital from June 2017 to June 2018 were selected as the study group. Healthy individuals $(n=30)$ who do not have CVDs were selected as the control group. All patients in the study group met the typical symptoms and diagnostic criteria of hypertension. All subjects were required to sign informed consent documents. Pregnant and lactating women were not included in this study. Fasting blood samples were collected and clinical data, such as sex, age, course of disease, BMI, smoking and drinking, dyslipidemia, diabetes mellitus, and diuretics, were recorded in detail.

\section{Inclusion criteria}

Patients who satisfied the following criteria were included in the study: (1) patients who fit the standard of hypertension diagnosis (refer to the diagnostic standard of hypertension expert consensus of China in 2010); (2) patients who fits the diagnostic criteria of AF recorded by electrocardiogram, $24 \mathrm{~h}$ dynamic electrocardiogram, and echocardiography; (3) patients who have no acute/chronic infective or autoimmune diseases; and (4) patients who are willing to sign an informed consent and can cooperate to complete the study.

\section{Exclusion criteria}

The exclusion criteria were as follows: (1) patients who do not fit the hypertension diagnostic criteria; (2) patients with gout, autoimmune, or inflammatory disease; (3) patients who have a history of heart surgery, respiratory diseases, thyroid diseases, and use of drugs that affect UA metabolism; and (4) unwillingness to participate.

\section{Sample collection}

All patients were asked to sign an informed consent form. In the morning, $5 \mathrm{ml}$ of fasting blood was collected from patients in each group for biological analysis. The serum was centrifuged $(15 \mathrm{~min}$, $\left.3,500 \times \mathrm{g}, 4^{\circ} \mathrm{C}\right)$ and stored in a refrigerator at $-80^{\circ} \mathrm{C}$ until use.

\section{ELISA assays}

Plasma samples were obtained from all groups and processed as previously described [14-15]. The concentrations of SUA, XO, High-Density Lipoprotein-Cholesterol (HDL-C), Low Density Lipoprotein-Cholesterol (LDL-C), Total Cholesterol (T-cho), Triglyceride (TG), and blood glucose were determined with the respective sensitive ELISA kits (R\&D Systems, Minneapolis, MN). The numbers of White Blood Cells (WBC), albumin, globulin, potassium, and creatinine were measured using Beckmann Kurt Uni Cel DxH 800 five-class hematology analyzer (purchased from Beckmann Kurt Commercial China Co., Ltd). The plasma levels of ROS biomarker (Nox-4) and inflammatory cytokines (Hs-CRP, IL$1 \beta$, IL- 8 , and TNF- $\alpha$ ) were determined using a competitive ELISA kit (R\&D Systems, Minneapolis, MN) following the manufacturer's instructions.

\section{Echocardiography}

Left Atrial Diameter (LAD), Interventricular Septal Thickness (IVST), Left Ventricular Posterior Wall Thickness (LVPWT), Left Ventricular End-Diastolic Dimension (LVEDD), and Left Ventricular Ejection Fraction (LVEF) were measured by DW-T6 trans thoracic doppler echocardiography (Beckmann Kurt Commercial China Co., Ltd).

\section{Statistical analysis}

All data were expressed as mean \pm SD. Student's t-test was performed to measure the differences between the groups. Fisher's protected least significant differences (PLSD) test of ANOVA was performed to analyze the quantitative data collected from the groups. A p value of $<0.05$ was used to denote significance.

\section{Results \\ Patient demographics}

According to the clinical manifestations, electrocardiogram, dynamic electrocardiogram, electrophysiological examination, and diagnostic criteria of hypertension, the patients were divided into hypertension with $\mathrm{AF}$ group (AF group), hypertension without $\mathrm{AF}$ group (non-AF group), and healthy control group (Table 1). The average age, BMI, course of hypertension, blood pressure, hyperlipidemia, and diabetes mellitus in the AF group were higher than those in the non$\mathrm{AF}$ and control groups $(\mathrm{P}<0.05$; Table 1$)$.

\section{Comparison of serum biochemicals among groups}

In comparison with the non-AF and control groups, the serum levels of $\mathrm{HDL}-\mathrm{c}, \mathrm{TG}$, and creatinine in the AF group were significantly increased $(\mathrm{P}<0.05)$, whereas the estimated glomerular filtration rate (eGFR) was significantly reduced $(\mathrm{P}<0.05)$, as shown in table 2 . Other blood biochemical parameters in the AF group showed altered tendencies, but no significant difference was observed among the different groups $(\mathrm{P}>0.05)$.

\section{Comparison of echocardiographic analysis among groups}

As shown in table 3, the echocardiographic parameters, such as LAD, IVST, LVPWT, and LVEF, in the AF group were significantly altered compared with those in the non-AF and control groups 
Table 1: Comparison of the general characteristics of patients in each group.

\begin{tabular}{|c|c|c|c|c|}
\hline Characteristic & Control $(n=30)$ & non-AF $(n=30)$ & $\operatorname{AF}(n=30)$ & $P$ value \\
\hline Male, n (\%) & $13(43.3 \%)$ & $11(36.7 \%)$ & $10(33.3 \%)$ & 0.04 \\
\hline Age & $36.45 \pm 6.34$ & $42.33 \pm 7.87$ & $48.67 \pm 9.34^{a}$ & 0.956 \\
\hline BMI $\left(\mathrm{kg} / \mathrm{m}^{2}\right)$ & $19.56 \pm 1.32$ & $22.32 \pm 1.88$ & $24.54 \pm 1.63^{a}$ & 0.048 \\
\hline Smoking, n (\%) & $6(20.0 \%)$ & $10(33.3 \%)$ & $11(36.7 \%)$ & 0.076 \\
\hline Drinking, n (\%) & $4(13.3 \%)$ & $6(20.0 \%)$ & $7(23.3 \%)$ & 0.647 \\
\hline Course of hypertension (years) & 0 & $12.22 \pm 9.35^{a}$ & $16.45 \pm 7.33^{a}$ & 0.014 \\
\hline $\mathrm{SBP}(\mathrm{mmHg})$ & $118.6 \pm 10.26$ & $141.4 \pm 15.34^{a}$ & $148.8 \pm 18.65^{a}$ & 0.023 \\
\hline $\mathrm{DBP}(\mathrm{mmHg})$ & $77.63 \pm 5.62$ & $85.52 \pm 7.82^{a}$ & $94.72 \pm 9.23^{a, b}$ & 0.031 \\
\hline With HLD, n (\%) & $0(0 \%)$ & $10(33.3 \%)^{a}$ & $17(56.6 \%)^{a, b}$ & 0.015 \\
\hline With DM, n (\%) & $0(0 \%)$ & $9(30.0 \%)^{a}$ & $18(60.0 \%)^{a, b}$ & 0.011 \\
\hline
\end{tabular}

Values are expressed in comparison with the control group. Data are presented as mean \pm SD. Differences among the groups were analyzed by Student's t-test. ${ }^{a} P<0.05$ versus control group; ${ }^{b} P<0.05$ versus non-AF group.

Abbreviations: BMI: Body Mass Index; BP: Systolic Blood Pressure; DBP: Diastolic Blood Pressure; HLD: Hyperlipidemia; DM: Diabetic Mellitus

Table 2: Comparison of serum biochemicals in each group.

\begin{tabular}{|c|c|c|c|c|}
\hline Characteristic & Control $(n=30)$ & non-AF $(n=30)$ & $A F(n=30)$ & P value \\
\hline WBC $\left(\times 10^{9} / \mathrm{L}\right)$ & $5.84 \pm 1.23$ & $6.32 \pm 1.36$ & $6.54 \pm 1.42$ & 0.623 \\
\hline Albumin (g/L) & $30.31 \pm 2.76$ & $33.63 \pm 3.53$ & $35.72 \pm 4.45$ & 0.867 \\
\hline Globulin (g/L) & $24.65 \pm 1.97$ & $25.74 \pm 2.87$ & $26.32 \pm 3.43$ & 0.962 \\
\hline Potassium (mmol/L) & $4.05 \pm 0.64$ & $4.14 \pm 0.65$ & $4.23 \pm 0.55$ & 0.762 \\
\hline T-cho (mmol/L) & $4.02 \pm 0.45$ & $4.36 \pm 0.71$ & $4.51 \pm 0.84$ & 0.431 \\
\hline HDL-c (mmol/L) & $1.09 \pm 0.13$ & $1.21 \pm 0.21$ & $1.25 \pm 0.32^{\mathrm{a}}$ & 0.034 \\
\hline LDL-c (mmol/L) & $2.38 \pm 0.27$ & $2.31 \pm 0.33$ & $2.28 \pm 0.54$ & 0.287 \\
\hline $\mathrm{TG}(\mathrm{mmol} / \mathrm{L})$ & $1.38 \pm 0.35$ & $1.45 \pm 0.46$ & $1.93 \pm 0.62^{\mathrm{a}, \mathrm{b}}$ & 0.037 \\
\hline Glucose (mmol/L) & $5.23 \pm 0.35$ & $5.37 \pm 0.61$ & $5.45 \pm 0.58$ & 0.126 \\
\hline Creatinine $(\mu \mathrm{mol} / \mathrm{L})$ & $87.36 \pm 6.75$ & $94 \pm 7.82$ & $120.34 \pm 15.24^{a, b}$ & 0.01 \\
\hline eGFR (mL/min/1.73m²) & $125.34 \pm 20.45$ & $116.57 \pm 13.92$ & $102.56 \pm 12.75^{\mathrm{a}}$ & 0.018 \\
\hline
\end{tabular}

Values are expressed relative to the control group. Data are presented as mean \pm SD. Differences between the two groups were analyzed by Student's t-test. a $P<0.05$ versus control group; ${ }^{b} P<0.05$ versus non-AF group.

Abbreviations: WBC: White Blood Cells; T-cho: Total Cholesterol; HDL-c: High Density Lipoprotein-Cholesterol; LDL-c: Low Density LipoproteinCholesterol; TG: Triglycerides; eGFR: Estimated Glomerular Filtration Rate

Table 3: Comparison of echocardiographic parameters in each group.

\begin{tabular}{|c|c|c|c|c|}
\hline Characteristic & Control $(n=30)$ & non-AF $(n=30)$ & $\operatorname{AF}(n=30)$ & P value \\
\hline IVST (mm) & $9.42 \pm 1.25$ & $9.73 \pm 1.33$ & $11.53 \pm 1.67^{a}$ & 0.041 \\
\hline LVPWT (mm) & $8.62 \pm 1.26$ & $9.02 \pm 1.43$ & $9.76 \pm 1.65$ & 0.047 \\
\hline $\mathrm{LAD}(\mathrm{mm})$ & $32.47 \pm 1.38$ & $35.63 \pm 1.45$ & $40.72 \pm 1.71^{\mathrm{a}}$ & 0.032 \\
\hline LVEDD (mm) & $44.21 \pm 1.18$ & $45.94 \pm 1.48$ & $47.66 \pm 1.54$ & 0.478 \\
\hline LVEF (\%) & $68.56 \pm 5.66$ & $52.43 \pm 5.42^{\mathrm{a}}$ & $40.85 \pm 6.45^{a, b}$ & 0.017 \\
\hline
\end{tabular}

Values are expressed in comparison with the control group. Data are presented as mean \pm SD. Differences between the two groups were analyzed by Student's t-test. ${ }^{a} P<0.05$ versus control group; ${ }^{b} P<0.05$ versus non-AF group.

Abbreviations: LAD: Left Atrial Diameter; IVST: Inter Ventricular Septal Thickness; LVPWT: Left Ventricular Posterior Wall Thickness; LVEDD: Left Ventricular End-Diastolic Dimension; LVEF: Left Ventricular Ejection Fraction 
$(\mathrm{P}<0.05)$. No statistical significance was found in the comparison of LVEDD among the groups ( $\mathrm{P}>0.05)$. UA, $\mathrm{XO}$, and Nox-4 expression was elevated in AF.

We performed ELISA to examine the plasma levels of UA, XO, and Nox-4 among the groups. The ELISA results showed the blood concentrations of UA, XO, and Nox-4 in the AF group significantly increase compared with those in the non-AF and control group $(\mathrm{P}<0.001$; Figures $1 \mathrm{~A}, \mathrm{~B}$, and $\mathrm{C}$, respectively).

\section{Inflammatory cytokines were increased in $\mathrm{AF}$}

ELISA results showed that the circulatory levels of inflammatory cytokines, such as Hs-CRP, IL- $1 \beta$, IL-8, and TNF- $\alpha$, significantly increased in the AF groups (Figure 2A-D) compared with the non$\mathrm{AF}$ and control group, and the difference was statistically significant $(\mathrm{P}<0.001$; Figures $2 \mathrm{~A}, \mathrm{~B}, \mathrm{C}$, and $\mathrm{D}$ respectively).

\section{Correlation between serum XO, Nox-4 and, inflammatory cytokines}

Pearson correlation analysis showed that the serum levels of XO in AF group were positively correlated with the blood concentrations of Nox-4 and inflammatory cytokines (Hs-CRP, IL-1 $\beta$, IL- 8 , and TNF- $\alpha$ ) with statistical significance $(\mathrm{P}<0.01)$, and the respective correlation coefficients were $r=0.99,0.91,0.96,0.95,0.97$.

Taken together, our result suggests that blood levels of XO significantly elevated ROS accumulation and inflammatory cytokines, thereby inducing AF formation in patients with hypertension.

\section{Discussion and Conclusion}

Among the different types of cardiac arrhythmia, AF is of great concern, because it remarkably contributes to cardiac mortality and morbidity. Patients with AF are at high risk of stroke, heart failure, and dementia [16]. Atrial systolic function is lost when AF occurs, and the blood is easily congests in the atrium to form a thrombus. When the thrombus falls off, it follows the blood to all parts of the body, leading to cerebral or arterial embolism [17]. Aging, gender, rheumatic heart disease, hypertension, congestive heart failure, hyperthyroidism, chronic kidney disease, and diabetes mellitus have been recognized as $\mathrm{AF}$ risk factors.

HUA is associated with cardiovascular and renal diseases and an independent predictor of premature death [18]. The prevalence of comorbidities increases with gout duration, and gout is associated
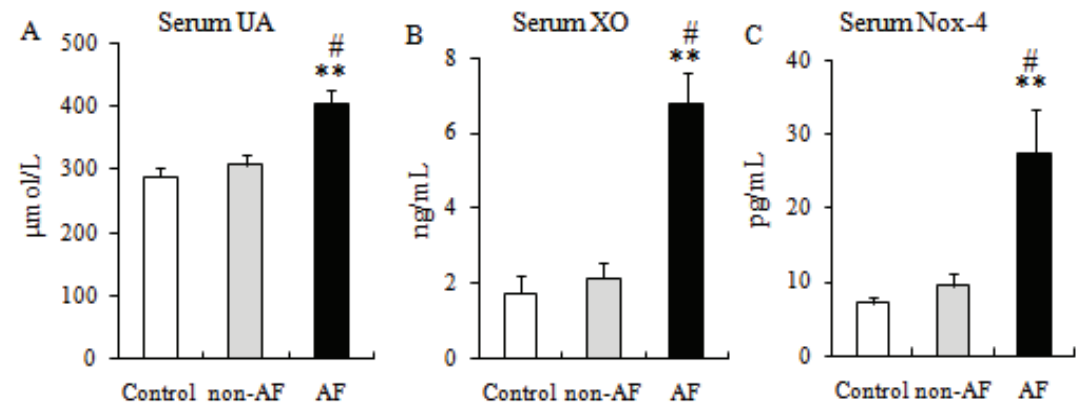

Figure 1: Uric acid, Xanthine Oxidase (XO), and NADPH oxidase-4 (Nox-4) expression was elevated in AF.

The blood levels of UA, XO, and Nox-4 were analyzed by ELISA. Values are expressed in comparison with the control group. Data are expressed as mean \pm SD. The quantitative data were analyzed with one-way ANOVA, followed by Fisher's PLSD test. The blood levels of UA (A), XO (B), and Nox$4(C)$ among the groups. ${ }^{* *} P<0.001$ versus control group, $\# P<0.01$ versus non-AF group.

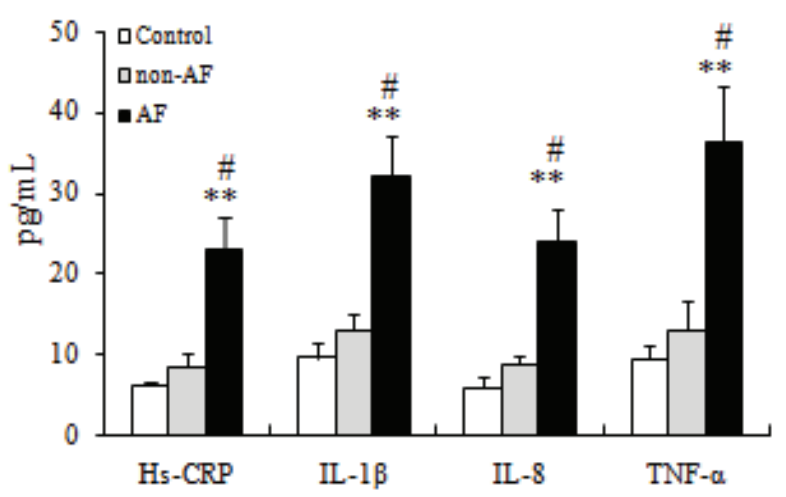

Figure 2: Inflammatory cytokines were increased in AF.

The plasma concentrations of inflammatory cytokines (Hs-CRP, IL-1 $\beta$, IL-8, and TNF- $\alpha$ ) were analyzed by ELISA. Values were expressed in comparison with the control group. Data were expressed as mean \pm SD. The quantitative data were analyzed with one-way ANOVA followed by Fisher's PLSD test. ${ }^{* *} P<0.001$ versus control group, $\# P<0.01$ versus non-AF group. 
with all components of the metabolic syndrome [19]. Hypertension is the one of the most frequent comorbidities of gout, and the prevalence of hypertension is $69.1 \%$ and $30.3 \%$ in gouty and nongouty patients, respectively [20]. The prevalence of abdominal obesity is higher for gouty than non-gouty patients at $62.9 \%$ versus $35.3 \%$ [21]. Additionally, the risk of gout increases with obesity [22]. BMI and waist circumference are positively associated with uricaemia, and adiposity causes HUA [23]. A recent meta-analysis study showed that Chronic Kidney Disease (CKD) of stage 3 in gout was estimated at $24 \%$ [24]. Reduced kidney function reduces rate excretion in urine and increases gout risk. In a cohort study among CKD patients, gout prevalence increased from $16.0 \%$ to $35.6 \%$ for CKD patients along with declining estimated glomerular filtration rate [25]. Among patients with hypertension and AF, age, BMI, blood pressure (systolic and diastolic), hyperlipidemia, diabetes mellitus, creatinine, and eGFR were significantly altered compared with those in the non-AF and healthy control groups (Tables 1,2)

The presence of hyperuricemia increases AF risk, particularly in patients who have undergone cardiac surgery, such as a Coronary Artery Bypass Graft (CABG). Thus, SUA level can increase the sensitivity and specificity in AF prediction after CABG surgery [26]. Letsas et al. [27] found that SUA level is remarkably correlated with the new onset of AF in a concentration-dependent manner. A metaanalysis of six cross-sectional and three cohort studies confirmed the association of high SUA with AF [28]. Pinelli et al. [29] found that in patients with heart failure, serum UA levels are correlated with LVEF, which is independent from other clinical determinants, thereby indicating that the detection of progressive hyperuricemia in these patients may be an indicator of deteriorating cardiac function. Cicero et al. [30] reported that high SUA is a risk factor for cardiac dysfunction, which can be evaluated by LVEF and by plasma B-type Natriuretic Peptide (BNP). Owczarek et al. [31] demonstrated that SUA concentration variability is closely associated with BMI, eGFR values, serum NT-pro BNP levels, LVEF, and the use of thiazide diuretics. In the present study, LVEF values were remarkably reduced in hypertension and $\mathrm{AF}$ patients compared with non-AF and healthy controls (Table 3).

Although the pathophysiology underlying AF remains unclear, accumulated evidence suggests the involvement of inflammation and oxidative stress [32-33]. HUA is associated with the incidence of paroxysmal or persistent $\mathrm{AF}$ and the risk of $\mathrm{AF}$ in post-cardiovascular surgery patients [33]. To investigate the link between HUA and AF, we refer to HUA-induced atrial remodeling. Both ionic channel and structural remodeling caused by HUA might explain AF's occurrence. Inhibition of XO and NADPH-oxidase or the use of antioxidants, along with the reduction in SUA levels prevent inflammation, might be useful. SUA is an independent predictor of CVD morbidity and mortality. The level of SUA is closely related to the severity of the disease and is an independent marker of adverse prognosis [34]. ROS plays an important role in $\mathrm{AF}$ and $\mathrm{CHF}$ occurrence and development. Oxidative stress during AF may play an important role in remodeling the atrial tissue [35]. Youn et al. [36] reported that the mechanisms of ROS production in human AF occurrence. They suggested that atrial oxidative stress during AF was caused by myocardial NADPH oxidase, nitric oxide synthase, and mitochondrial oxidases, which were associated with the electrical remodeling observed in AF. These findings suggest that oxidative stress may directly or indirectly regulate the metabolism of atrial muscle and functional properties of the atrium, thereby leading to atrial remodeling. Oxidative stress is closely associated with the pathogenesis of $\mathrm{AF}$ and hypertension results from overproduction of ROS. Long-term exposure to oxidative stress in hypertension induces chronic inflammation, vascular cell proliferation, and/or apoptosis in cardiovascular system, leading to formation and progression of disease states in this system. Indeed, markers for oxidative stress are over expressed in patients with hypertension and AF, suggesting that increased ROS may be primarily responsible for the development of AF. In the present study, serum levels of UA and XO in patients with hypertension and AF substantially elevated the expression levels of Nox- 4 and accelerated AF progression (Figure 1).

In the current study, direct measurement of ROS was not performed and this may consider a limitation associated with the study, which will need to be addressed in future work. Previously we reported that XO markedly induced the accumulations of ROS in adipose [9] and colon [14]. In the presents study we analyzed the expressions ROS marker (NADPH oxidase-4, Nox-4) using ELISA method. The results demonstrated that in AF patients high levels of XO significantly increased the circulatory levels of Nox-4. Therefore, we consume that $\mathrm{XO}$ significantly induced ROS production in AF patients.

The left appendage tissues obtained from AF patients showed more prominent presence of CD45-reactive cells, CD68-positive macrophages, and less CD3-positive $\mathrm{T}$ cells compared with tissues obtained from patients with a sinus rhythm [37]. In the atrium, inflammation induced its enlargement and the development of atrial fibrosis. Atrial fibrosis promoted AF by interrupting the continuity of fiber bundles, thereby causing atrial conduction disturbance [38]. Chao et al. [39] found that hs-CRP expression and insulin resistance were higher, and the LA diameter was large in patients with high SUA level. Increased inflammation and insulin resistance may be involved in the relationship between high SUA level and LA size. Mice treated with angiotensin-II showed an increase of neutrophil infiltration in their atrial tissue, which depends on CD11b and CD18 integrins, because angiotensin II stimulates the production of pro-inflammatory cytokines, such as IL-6, IL-8, and TNF- $\alpha$, through the activation of angiotensin I receptors [40]. Although the relationship between $\mathrm{UA}$ and the RAAS system in cardiac atrial myocytes has never been tested, UA could stimulate the circulating and local RAAS system in the cardiovascular system. In accordance with these reports, we also demonstrated that the circulatory levels of inflammatory cytokines (Hs-CRP, IL-1 $\beta$, IL-8, and TNF- $\alpha$ ) were remarkably elevated in hypertension and AF patients, and SUA levels were closely associated with the levels of these cytokines (Figure 2).

In conclusion, SUA is independently associated with the occurrence of AF. The accumulation of UA inside atrial cardiomyocytes might cause ionic and structural remodeling of the atria, which is referred to as atrial remodeling. In this study, the differences of LAD, IVST, LVPWT, and LVEF between the AF and non-AF group were significant $(\mathrm{P}<0.05)$ and may be related to the corresponding increase of $U A$ and XO levels. The differences of age, BMI, and eGFR between the AF and non-AF group were statistically significant $(\mathrm{P}<0.05)$ and may be related to the increase of $\mathrm{BMI}$ and renal function. More importantly, $\mathrm{XO}$ activity in the AF group remarkably increased, thereby suggesting that SUA levels increased accordingly and further leading to the production of ROS (Nox-4), the activation of inflammatory cytokines (Hs-CRP, IL-1 $\beta$, IL- 8 , and TNF- $\alpha$ ), and the occurrence of AF. More studies are needed to obtain a more comprehensive understanding of $\mathrm{AF}$ pathophysiology and the measures to prevent it.

\section{Acknowledgments}

We thank Dr. Kyosuke Takeshita for carefully reading and applying changes to our manuscript. 


\section{Funding and Disclosure}

The current study was supported by a grant from the Xinjiang Uygur Autonomous Region Natural Science Foundation Program (grant no. 2018D01C134). No duality of interest during the writing of this manuscript was declared by the authors.

\section{Contribution Statement}

MA, MY, and ZZZ contributed to the conceptualization and formulation of the experimental design and the interpretation of the experimental results. MA, AM, MY, WMZ, AA, YZ, TM, and XZ performed the experiments and analyzed the data.MA, MY, and WMZ wrote the manuscript. MA, MY, and ZZZ ensured the integrity of the entire work. This manuscript was revised by its authors. All authors decided to submit this version for publication.

\section{References}

1. Ogunsua AA, Shaikh AY, Ahmed M, McManus DD (2015) Atrial Fibrillation and Hypertension: Mechanistic, Epidemiologic, and Treatment Parallels. Methodist Debakey Cardiovasc J 11: 228-234.

2. Shenasa M, Shenasa H (2017) Hypertension, left ventricular hypertrophy, and sudden cardiac death. Int J Cardiol 237: 60-63.

3. Lin GM, Li YH, Zheng NC, Lai CP, Lin CL, et al. (2013) Serum uric acid as an independent predictor of mortality in high-risk patients with obstructive coronary artery disease: a prospective observational cohort study from the ET-CHD registry, 1997-2003. J Cardiol 61: 122-127.

4. Doehner W, Jankowska EA, Springer J, Lainscak M, Anker SD (2016) Uric acid and xanthine oxidase in heart failure - Emerging data and therapeutic implications. Int J Cardiol 213: 15-19.

5. Yoshizawa T, Niwano S, Niwano H, Tamaki H, Nakamura $\mathrm{H}$, et al. (2018) Antiremodeling Effect of Xanthine Oxidase Inhibition in a Canine Model of Atrial Fibrillation. Int Heart J 59: 1077-1085.

6. Bove M, Cicero AFG, Borghi C (2017) The Effect of Xanthine Oxidase Inhibitors on Blood Pressure and Renal Function. Curr Hypertens Rep 19: 95.

7. Ren X, Wang X, Yuan M, Tian C, Li H, et al. (2018) Mechanisms and Treatments of Oxidative Stress in Atrial Fibrillation. Curr Pharm Des 24: 3062-3071.

8. Liang X, Zhang Q, Wang X, Yuan M, Zhang Y, et al. (2018) Reactive oxygen species mediated oxidative stress links diabetes and atrial fibrillation. Mol Med Rep 17: 4933-4940.

9. Yisireyili M, Hayashi M, Wu H, Uchida Y, Yamamoto K, et al. (2017) Xanthine oxidase inhibition by febuxostat attenuates stress-induced hyperuricemia, glucose dysmetabolism, and prothrombotic state in mice. Sci Rep 7: 1266.

10. Harada M, Van Wagoner DR, Nattel S (2015) Role of inflammation in atrial fibrillation pathophysiology and management. Circ J 79: 495-502.

11. Hu YF, Chen YJ, Lin YJ, Chen SA (2015) Inflammation and the pathogenesis of atrial fibrillation. Nat Rev Cardiol 12: 230-243.

12. Serban RC, Balan Al, Perian M, Pintilie I, Somkereki C, et al. (2019) Atrial electrical remodeling induced by chronic ischemia and inflammation in patients with stable coronary artery disease. Chin J Physiol 62: 11-16.

13. Cingolani HE, Plastino JA, Escudero EM, Mangal B, Brown J, et al. (2006) The effect of xanthine oxidase inhibition upon ejection fraction in heart failure patients: La Plata Study. J Card Fail 12: 491498.
14. Yisireyili M, Uchida Y, Yamamoto K, Nakayama T, Cheng XW, et al. (2018) Angiotensin receptor blocker irbesartan reduces stressinduced intestinal inflammation via AT1a signaling and ACE2dependent mechanism in mice. Brain Behav Immun 69: 167-179.

15. Aizezi M, Yisireyili M, Zhang W, Alimujiang A, Zhang H, et al. (2019) Analysis of the Clinical Effects and Expression Levels of Inflammatory Cytokines in Type B AD Patients after TEVAR Therapy. J Clin Case Stu 4.

16. Zimetbaum P (2017) Atrial Fibrillation. Ann Intern Med 166: ITC33ITC48.

17. Kallistratos MS, Poulimenos LE, Manolis AJ (2018) Atrial fibrillation and arterial hypertension. Pharmacol Res 128: 322-326.

18. Sharaf El Din UAA, Salem MM, Abdulazim DO (2017) Uric acid in the pathogenesis of metabolic, renal, and cardiovascular diseases: $A$ review. J Adv Res 8: 537-548.

19. Bardin T, Richette P (2017) Impact of comorbidities on gout and hyperuricaemia: an update on prevalence and treatment options. BMC Med 15: 123.

20. Choi HK, Ford ES, Li C, Curhan G (2007) Prevalence of the metabolic syndrome in patients with gout: the Third National Health and Nutrition Examination Survey. Arthritis Rheum 57: 109-115.

21. Choi HK, Atkinson K, Karlson EW, Curhan G (2005) Obesity, weight change, hypertension, diuretic use, and risk of gout in men: the health professionals follow-up study. Arch Intern Med 165: 742-748.

22. Lyngdoh $T$, Vuistiner $P$, Marques-Vidal $P$, Rousson $V$, Waeber $G$, et al. (2012) Serum uric acid and adiposity: deciphering causality using a bidirectional Mendelian randomization approach. PLoS One 7: e39321.

23. Xu M, Su J, Hao J, Zhong N, Zhang Z, et al. (2018) Positive association between serum uric acid and bone mineral density in Chinese type 2 diabetes mellitus stratified by gender and BMI. J Bone Miner Metab 36: 609-619.

24. Roughley MJ, Belcher J, Mallen CD, Roddy E (2015) Gout and risk of chronic kidney disease and nephrolithiasis: meta-analysis of observational studies. Arthritis Res Ther 17: 90.

25. Jing J, Kielstein JT, Schultheiss UT, Sitter T, Titze SI, et al. (2015) Prevalence and correlates of gout in a large cohort of patients with chronic kidney disease: the German Chronic Kidney Disease (GCKD) study. Nephrol Dial Transplant 30: 613-621.

26. Memetoglu ME, Kehlibar T, Yılmaz M, Günay R, Arslan Y, et al. (2015) Serum uric acid level predicts new-onset atrial fibrillation after coronary artery bypass graft operation. Eur Rev Med Pharmacol Sci 19: 784-789.

27. Letsas KP, Korantzopoulos P, Filippatos GS, Mihas CC, Markou V, et al. (2010) Uric acid elevation in atrial fibrillation. Hellenic J Cardiol 51: 209-213.

28. Tamariz L, Hernandez F, Bush A, Palacio A, Hare JM (2014) Association between serum uric acid and atrial fibrillation: a systematic review and meta-analysis. Heart Rhythm 11: 1102-1108.

29. Pinelli M, Bindi M, Filardo FP, Moroni F, Castiglioni M (2007) Serum uric acid levels correlate with left ventricular ejection fraction and systolic pulmonary artery pressure in patients with heart failure. Recenti Prog Med 98: 619-623. 
30. Cicero AF, Rosticci M, Fogacci F, Grandi E, D'Addato S, et al. (2017) High serum uric acid is associated to poorly controlled blood pressure and higher arterial stiffness in hypertensive subjects. Eur J Intern Med 37: 38-42.

31. Owczarek AJ, Choręza P, Arabzada H, Chudek J, Wojnicz R (2018) Kidney Function, Nutritional Status, and the Left Ventricle Dysfunction Are Associated with Serum Uric Acid Levels in Patients with Heart Failure with Reduced Ejection Fraction. Ann Clin Lab Sci 48: 608-613.

32. Karam BS, Chavez-Moreno A, Koh W, Akar JG, Akar FG (2017) Oxidative stress and inflammation as central mediators of atrial fibrillation in obesity and diabetes. Cardiovasc Diabetol 16: 120.

33. Zakkar M, Ascione R, James AF, Angelini GD, Suleiman MS (2015) Inflammation, oxidative stress and postoperative atrial fibrillation in cardiac surgery. Pharmacol Ther 154: 13-20.

34. Tamariz L, Harzand A, Palacio A, Verma S, Jones J, et al. (2011) Uric acid as a predictor of all-cause mortality in heart failure: a metaanalysis. Congest Heart Fail 17: 25-30.
35. Kazakov A, Hall RA, Werner C, Meier T, Trouvain A, et al. (2018) Raf kinase inhibitor protein mediates myocardial fibrosis under conditions of enhanced myocardial oxidative stress. Basic Res Cardiol 113: 42.

36. Youn JY, Zhang J, Zhang Y, Chen H, Liu D, et al. (2013) Oxidative stress in atrial fibrillation: an emerging role of NADPH oxidase. J Mol Cell Cardiol 62: 72-79.

37. Yamashita T, Sekiguchi A, Iwasaki YK, Date T, Sagara K, et al. (2010) Recruitment of immune cells across atrial endocardium in human atrial fibrillation. Circ J 74: 262-270.

38. Thanigaimani S, Lau DH, Agbaedeng T, Elliott AD, Mahajan R, et al. (2017) Molecular mechanisms of atrial fibrosis: implications for the clinic. Expert Rev Cardiovasc Ther 15: 247-256.

39. Chao TF, Hung CL, Chen SJ, Wang KL, Chen TJ, et al. (2013) The association between hyperuricemia, left atrial size and new-onset atrial fibrillation. Int J Cardiol 168: 4027-4032.

40. Friedrichs K, Adam M, Remane L, Mollenhauer M, Rudolph V, et al. (2014) Induction of atrial fibrillation by neutrophils critically depends on CD11b/CD18 integrins. PLoS One 9: e89307. 